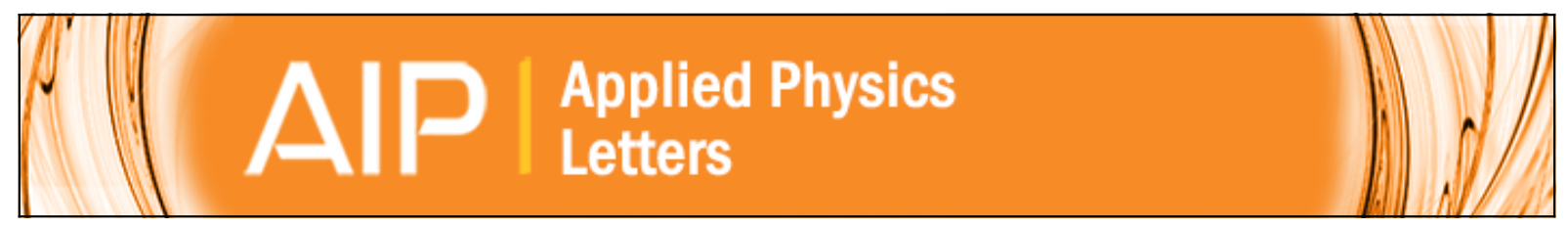

\title{
Enhanced parametric processes in binary metamaterials
}

Maxim V. Gorkunov, llya V. Shadrivov, and Yuri S. Kivshar

Citation: Applied Physics Letters 88, 071912 (2006); doi: 10.1063/1.2168755

View online: http://dx.doi.org/10.1063/1.2168755

View Table of Contents: http://scitation.aip.org/content/aip/journal/apl/88/7?ver=pdfcov

Published by the AIP Publishing

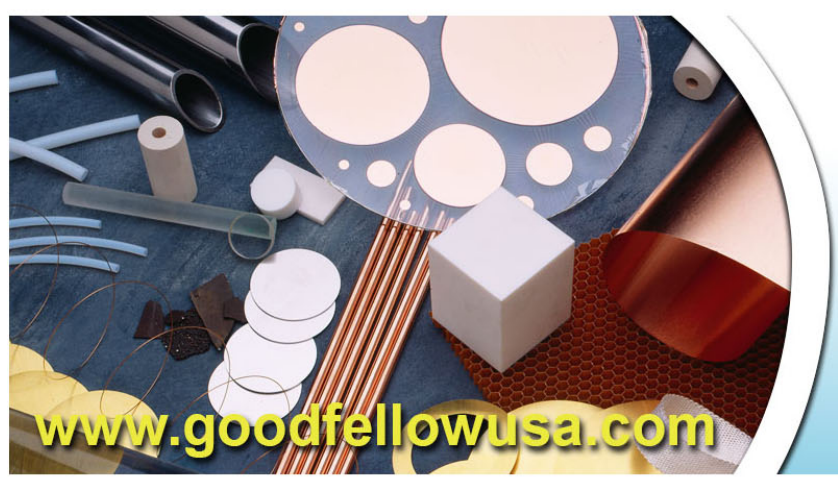

BOOdFEl|OU metals • ceramics $\cdot$ polymers composites $\cdot$ compounds $\cdot$ glasses

Save $5 \% \cdot$ Buy online 70,000 products $\cdot$ Fast shipping 


\title{
Enhanced parametric processes in binary metamaterials
}

\author{
Maxim V. Gorkunov \\ Nonlinear Physics Centre, Research School of Physical Sciences and Engineering, Australian National \\ University, Canberra Australian Capital Territory 0200, Australia and Institute of Crystallography, Russian \\ Academy of Science, Moscow 119333, Russia
}

Ilya V. Shadrivov and Yuri S. Kivshar

Nonlinear Physics Centre, Research School of Physical Sciences and Engineering, Australian National University, Canberra Australian Capital Territory 0200, Australia

(Received 7 October 2005; accepted 5 January 2006; published online 14 February 2006)

\begin{abstract}
We suggest double-resonant (binary) metamaterials composed of two types of magnetic resonant elements, and demonstrate that in the nonlinear regime such metamaterials provide unique possibilities for phase-matched parametric interaction and enhanced second-harmonic generation. (C) 2006 American Institute of Physics. [DOI: 10.1063/1.2168755]
\end{abstract}

Extensive studies of microwave properties of composite metallic structures led to the experimental demonstration of left-handed metamaterials ${ }^{1}$ suggested a long time ago. ${ }^{2}$ Such metamaterials are created by resonant magnetic elements and operate for wavelengths much larger than the structural period, allowing for the macroscopic effective medium description. The parameters of the effective medium depend on the microscopic structure of the metallic composites. Moreover, the nonlinear response of the metamaterial can become substantial, ${ }^{3-5}$ and their transmission characteristics can be effectively controlled by external fields. ${ }^{6-8}$

Nonlinearities of metamaterials suggest their novel applications such as frequency conversion, 5,9 tunable transmission, ${ }^{8}$ second-harmonic imaging, ${ }^{10}$ nonlinear beam focusing, and soliton propagation, ${ }^{11}$ etc. In contrast to nonlinear optical media, composite metamaterials possess nonlinear magnetic responses that can be engineered by inserting nonlinear elements (e.g., diodes) into the resonant conductive elements. 3,4

In this letter we suggest a novel type of composite metamaterial with a double-resonant response and demonstrate that in the nonlinear regime such binary metamaterials are ideally suited for the first observation of the enhanced phase-matched parametric interaction and second-harmonic generation (SHG). Indeed, the quadratic nonlinear magnetic susceptibility is proportional to a product of linear magnetic susceptibilities at the frequencies of interacting waves. For conventional single-resonant nonlinear metamaterials, the magnetic susceptibility of the fundamental wave is relatively large, since it corresponds to the backward wave near the resonance ${ }^{9}$ while the susceptibility of the second-harmonic wave is rather small. In the metamaterial with several resonances, it is possible to enhance the nonlinear response, so that both linear susceptibilities of interacting waves can become large.

To create a double-resonant metamaterial we suggest the mixing of two types of resonant conductive elements (RCEs) with different resonant frequencies, as shown schematically in Fig. 1 for the structure consisting of two lattices of different split-ring resonators. Metamaterial structures with two resonances were studied experimentally, ${ }^{12}$ as well as theoretically ${ }^{13}$ within the quasistatic approximation for oneand two-dimensional arrays only. Therefore, as a first step, we analyze linear properties of the three-dimensional binary metamaterial. For large wavelengths, each RCE can be described as a resonant circuit (see, e.g., Refs. 14 and 15) characterized by self-inductance $L$, capacitance $C$, and resistance $R$. We assume that the metamaterial consists of two types of RCEs of the same shape (i.e., with the same $L$ and $R$ ), but with different capacitances $C_{1}$ and $C_{2}$, and, thus, different resonant frequencies.

An external homogeneous magnetic field $H_{0}$ applied perpendicular to the RCE planes and oscillating with the frequency $\omega$ induces the currents $I_{1}$ and $I_{2}$ in the resonators of the corresponding type, which can be found from the impedance matrix equation

$$
\mathcal{E}=Z_{1,2} I_{1,2}+\Xi I_{2,1},
$$

where $Z_{1,2}=Z_{1,2}^{(0)}-i \omega L_{11}$ and $\Xi=-i \omega L_{12} ; \mathcal{E}=i \mu_{0} \omega S H_{0}$ is the electromotive force; $S$ is the RCE area; $Z_{\alpha}^{0}(\omega)=-i \omega L$ $+i\left(\omega C_{\alpha}\right)^{-1}+R$ is the self-impedance of an RCE of the type $\alpha$; $N_{\alpha}$ denotes the set of RCE position indices of the type $\alpha$; and $M_{n^{\prime} n}$ are the mutual inductances. The effective inductances are

$$
L_{11}=\sum_{\substack{n, n^{\prime} \in N_{1}, n^{\prime} \neq n}} M_{n^{\prime} n} ; \quad L_{12}=\sum_{\substack{n \in N_{1}, n^{\prime} \in N_{2},}} M_{n^{\prime} n} .
$$

Solving the set of Eqs. (1) with respect to the currents, we obtain the magnetization of the metamaterial,

$$
M=\frac{1}{2} n S\left(I_{1}+I_{2}\right)=n S^{2} \mu_{0} \mathcal{K} H_{0},
$$

where $\mathcal{K}=i \omega\left(Z_{1}+Z_{2}-2 \Xi\right) / 2\left(Z_{1} Z_{2}-\Xi^{2}\right)$, and $n=\left(a^{2} b\right)^{-1}$ is the total volume density of the RCEs. Using the general

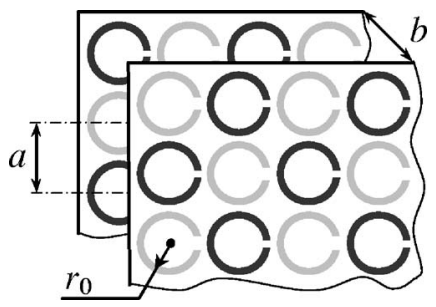

FIG. 1. Schematic structure of binary metamaterials with resonant magnetic elements of two types (black and gray) 

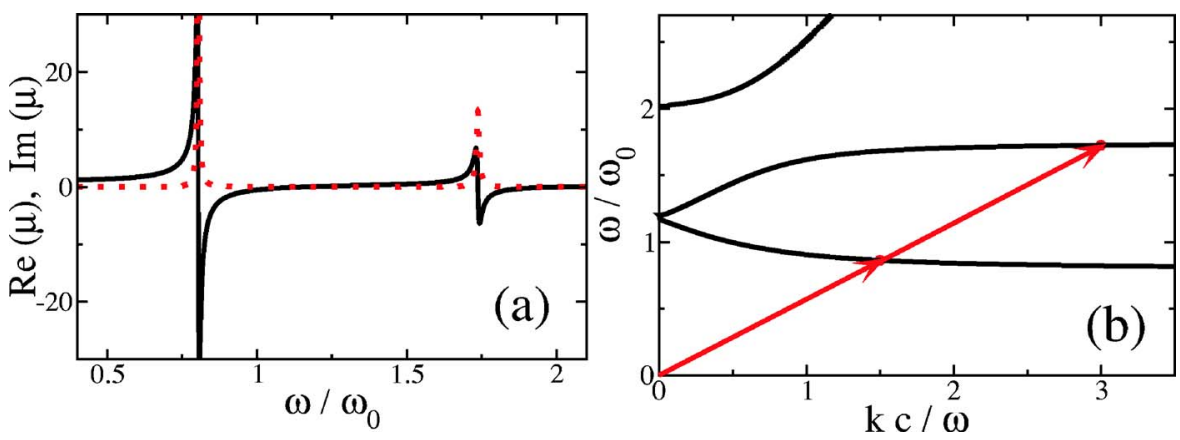

FIG. 2. (Color online) (a) Real (solid) and imaginary (dashed) parts of magnetic permeability of the binary metamaterial. (b) Spectrum of electromagnetic waves. The arrows show the perfectly phase-matched secondharmonic generation. relation for magnetic induction of media in the external field, $B=\mu_{0}\left(H_{0}+2 / 3 M\right)$ (see Ref. 14 for details) and the definition of the magnetic susceptibility $\chi, M=\chi H$, we calculate the magnetic permeability $\mu$,

$$
\mu(\omega)=1+\chi=1+\frac{\mu_{0} n S^{2}}{\mathcal{K}^{-1}-\mu_{0} n S^{2} / 3} .
$$

In the case $C_{1}=C_{2}$, the result (4) reduces to that obtained previously for single-resonant structures. ${ }^{14}$

In Fig. 2(a), we plot the permeability versus frequency for typical parameters: RCE radius $r_{0}=2 \mathrm{~mm}$, wire thickness $l=0.1 \mathrm{~mm}$, which gives self-inductance $L=8.36 \mathrm{nHn}$ (see Ref. 14). To obtain RCEs of the type 1 with the resonant frequency of $\omega_{01}=6 \pi \times 10^{9} \mathrm{rad} / \mathrm{s} \quad\left(\nu_{0}=3 \mathrm{GHz}\right)$, we take $C_{1}=0.34 \mathrm{pF}$. The resonance frequency of the type 2 RCEs is chosen as $\omega_{02}=X \omega_{01}$ with $X=1.75$, i.e., $C_{2}=C_{1} / X^{2}$. The lattice constants are $a=2.1 r_{0}$ and $b=0.5 r_{0}$. The RCE quality factor, $Q=\omega_{01} L / R$, can reach the values up to $10^{3} .{ }^{1}$ However, by inserting diodes this value may decrease, and therefore we take $Q=300$.

Figure 2(a) confirms that indeed in such structures there exist two resonances and two frequency ranges with negative magnetic permeability. Positions of the macroscopic resonances are shifted from the resonant frequencies of individual RCEs; the shift is not the same for two resonances, and the resulting ratio of the resonant frequencies is about 2.17 .

Nonlinear metamaterials can be created by inserting nonlinear elements. In order to obtain a material with low resistive losses, it is preferable to use variable capacitance insertions, varactor diodes. ${ }^{3}$ We assume that the capacitance of RCEs (both linear and nonlinear) is determined by varactors, and the difference between two types of resonators arises due to different varactors.

A general expression for the voltage drop on a varactor can be written in the form ${ }^{5,6}$

$$
U(t)=I(t) R_{\mathrm{ins}}[U(t)]+\frac{1}{C_{\mathrm{ins}}[U(t)]} \int_{-\infty}^{t} I\left(t^{\prime}\right) d t^{\prime},
$$

and it can be simplified in the limit of low current and low voltage. We assume that resistance is constant, while capacitance can be expanded into a Taylor series, $C_{\text {ins }}(U) \simeq C(1$ $+\gamma U$ ). The corresponding solution of Eq. (5) gives a linear capacitive contribution to the impedance as well as weak quadratic nonlinearity.

For the three-wave parametric processes, we write $I_{\alpha}(t)=\sum_{\nu=1}^{3} I_{\alpha}\left(\omega_{\nu}\right) \exp \left(-i \omega_{\nu} t\right)+$ c.c. $, \quad \omega_{1}=\omega_{2}+\omega_{3}, \quad$ and the nonlinear analog of Eq. (1) takes the form

$$
\begin{aligned}
\mathcal{E}\left(\omega_{1,2}\right)= & Z_{1,2}\left(\omega_{1,2}\right) I_{1,2}\left(\omega_{1,2}\right)+\Xi\left(\omega_{1,2}\right) I_{2,1}\left(\omega_{1,2}\right) \\
& +\frac{\gamma_{1,2}}{C_{1,2}^{2} \omega_{2} \omega_{3}} I_{1,2}\left(\omega_{2}\right) I_{1,2}\left(\omega_{3}\right) .
\end{aligned}
$$

Since the nonlinear part of the capacitance is much smaller than the linear one, we apply an iterative procedure and use linear currents calculating nonlinear contribution to obtain

$$
\begin{aligned}
\chi^{(2)}\left(\omega_{1} ; \omega_{2}, \omega_{3}\right)= & \frac{4 i \chi\left(\omega_{1}\right) \chi\left(\omega_{2}\right) \chi\left(\omega_{3}\right)}{\mu_{0} S^{3} n^{2} \omega_{1} \omega_{2} \omega_{3}} \\
& \times \sum_{\alpha} \frac{\gamma_{\alpha}}{C_{\alpha}^{2}} A_{\alpha}\left(\omega_{1}\right) A_{\alpha}\left(\omega_{2}\right) A_{\alpha}\left(\omega_{3}\right),
\end{aligned}
$$

where

$$
A_{1,2}(\omega)=\frac{Z_{2,1}(\omega)-\Xi(\omega)}{Z_{1}(\omega)+Z_{2}(\omega)-2 \Xi(\omega)},
$$

characterizes the relative contribution from the currents, $I_{1}(\omega)$ and $I_{2}(\omega)$, to the total magnetization of the metamaterial, and $A_{1}+A_{2}=1$. In the limit of identical varactors in both sublattices, i.e., $C_{1}=C_{2}$ and $\gamma_{1}=\gamma_{2}$, Eq. (7) coincides with the results obtained in Ref. 3 .

We note that the zeros of the denominator in Eq. (8) are canceled out by zeros of linear susceptibility in the numerator and they do not lead to any increase of the nonlinear response. At the same time, the resonant poles of the linear magnetic susceptibilities in Eq. (7) lead to a dramatic increase of nonlinear interaction when the wave frequencies approach resonances. Clearly, the effect is stronger when all the waves are close to the magnetic resonances of the metamaterial. Therefore, the binary metamaterial provides an unique possibility for realizing this general concept, as we show below for the example of SHG.

As has been shown recently, ${ }^{9}$ SHG in the media with negative refraction differs from the conventional scheme. In particular, it is possible to satisfy the phase-matching conditions for counterpropagating waves. As a result, a semiinfinite sample of a nonlinear quadratic metamaterial operates as a frequency converting mirror reflecting radiation with the double frequency of the incident wave. Remarkably, in the lossless regime the conversion efficiency is close to $100 \%$. In a more realistic case of a finite-size metamaterial slab, high efficiency is possible for the slabs of several tens of wavelengths.

For the double-resonant medium, first we analyze the spectrum of electromagnetic waves, $\omega(k)$. We consider the waves with the magnetic field perpendicular to the planes of resonators and assume that the electric component of the metamaterial generates a plasmaiike dielectric response, 


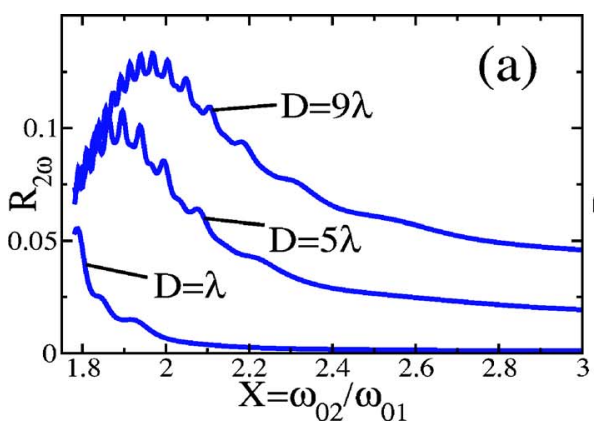

(a)

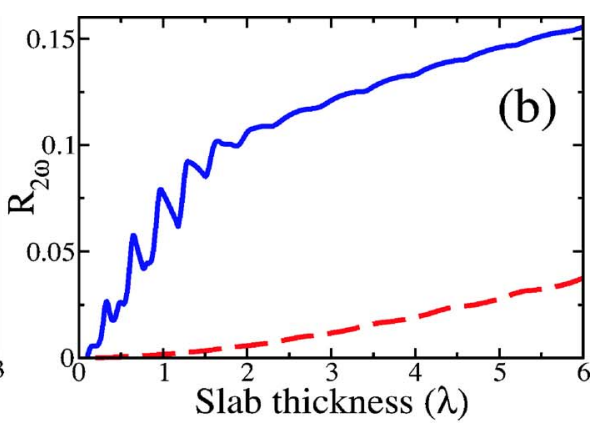

FIG. 3. (Color online) (a) Reflection coefficient of the second harmonics as function of resonant frequency ratio $X$, for different slab thicknesses $D$. (b) Maximum reflection coefficient of the second harmonics (solid) and reflection coefficient at $X=3$ (dashed) as function of slab thickness. $\varepsilon(\omega)=1-\omega_{\mathrm{p}}^{2} / \omega^{2}$, where the plasma frequency $\omega_{\mathrm{p}}=1.2 \omega_{0}$ is selected between two magnetic resonances. The wave spectrum has three branches, as shown in Fig. 2(b). Two branches, which are close to the magnetic resonances, correspond to large wave numbers. Importantly, we can find the points of the exact phase matching between fundamental and second-harmonic waves, for both waves close to the resonances.

We consider the case of normal incident wave propagating along the $z$ axis, and present the magnetic field inside the sample using slowly varying amplitudes

$$
\begin{aligned}
H(z, t)= & a_{1}^{+}(z) e^{-i k z-i \omega t}+a_{2}^{+}(z) e^{-2 i k z-2 i \omega t}+a_{1}^{-}(z) e^{i k z-i \omega t} \\
& +a_{2}^{-}(z) e^{2 i k z-2 i \omega t}+\text { c.c. },
\end{aligned}
$$

where $k=\omega / c \sqrt{\varepsilon(\omega) \mu^{\prime}(\omega)}$ (as usual $\mu=\mu^{\prime}+i \mu^{\prime \prime}$ ), the phase mismatch $\Delta=\varepsilon(\omega) \mu^{\prime}(\omega)-\varepsilon(2 \omega) \mu^{\prime}(2 \omega)$ is assumed to be small. The coupled-mode equations for the amplitudes $a_{1}^{+}$ and $a_{2}^{+}$are written in the form

$$
\begin{aligned}
& \frac{d a_{1}^{+}}{d \zeta}+\kappa_{1} a_{1}^{+}=i \sigma_{1} a_{2}^{+} a_{1}^{+*}, \\
& \frac{d a_{2}^{+}}{d \zeta}+\kappa_{2} a_{2}^{+}=-i \sigma_{2} a_{1}^{+2},
\end{aligned}
$$

where we use the notations $\kappa_{1}=\mu^{\prime \prime}(\omega) \varepsilon(\omega)^{1 / 2} / 2 \mu^{\prime}(\omega)^{1 / 2}$, $\kappa_{2}=\left[i \Delta-\varepsilon(2 \omega) \mu^{\prime \prime}(2 \omega)\right]\left[\varepsilon(\omega) \mu^{\prime}(\omega)\right]^{-1 / 2}$,

$\sigma_{1}=0.5\left[\varepsilon(\omega) / \mu^{\prime}(\omega)\right]^{1 / 2} \chi^{(2)}(\omega ; 2 \omega,-\omega)$,

$\sigma_{2}=\varepsilon(2 \omega)\left[\mu^{\prime}(\omega) \varepsilon(\omega)\right]^{-1 / 2} \chi^{(2)}(2 \omega ; \omega, \omega)$, and $\zeta=\omega z / c$ is the dimensionless coordinate. Equations for the amplitudes $a_{1}^{-}$ and $a_{2}^{-}$are the same as equations for $a_{1}^{+}$and $a_{2}^{+}$, except for the opposite signs of the spatial derivatives. We solve these equations numerically with appropriate boundary conditions and obtain the dependence of the second-harmonic ( $\mathrm{SH}$ ) reflection coefficient, i.e., the ratio of the reflected energy flux of the SH to the incident wave, as a function of the ratio of the two resonant frequencies $X$, shown in Fig. 3(a) for three slab thicknesses. Calculating the results shown in Fig. 3(a), we were adjusting the frequency of the incident wave to satisfy the phase-matching conditions. Large $X$ correspond to the nonresonant limit, when the $\mathrm{SH}$ field is not in resonance. Decreasing $X$ we drive both fundamental and $\mathrm{SH}$ waves closer to the magnetic resonances, and the conversion rate increases. At the same time, losses become stronger, and finally they dominate, suppressing SHG efficiency. For small relative shifts (below $X=1.75$ ), the phase matching cannot be archived. The incident field amplitude and nonlinear coefficients $\alpha_{1}=\alpha_{2}$, were chosen in such a way that maximum nonlinear modulation in the simulations was $\chi^{(2)}(\omega ; 2 \omega$, $-\omega) H_{\omega}<0.2$. Such modulation is expected in resonant nonlinear processes, since even in realistic nonresonant cases, ${ }^{5}$ the nonlinear modulation of 0.01 was created by the external magnetic fields with amplitudes less than $1 \mathrm{~A} / \mathrm{m}$. Our results demonstrate that for a one-wavelength-thick slab, the SHG enhancement due to the second resonance can become larger by at least one order of magnitude. The decrease of losses would allow increasing the efficiency.

Dependence of the maximum reflection coefficient of the $\mathrm{SH}$ wave and reflection coefficient in the nonresonant case $(X=3)$ on the slab thickness is shown in Fig. 3(b). One can see that the major relative increase of the SHG process in resonance, compared to the nonresonant case, is observed for thin nonlinear slabs.

In conclusion, we have suggested double-resonant metamaterials for the study of phase-matched parametric interactions in composite nonlinear media. In particular, we have analyzed a composite structure consisting of two types of resonant magnetic elements, and demonstrated that such a binary resonant structure can enhance significantly the second-harmonic generation.

${ }^{1}$ R. A. Shelby, D. R. Smith, and S. Schultz, Science 292, 77 (2001).

${ }^{2}$ V. G. Veselago, Sov. Phys. Solid State 8, 2854 (1967).

${ }^{3}$ M. Lapine, M. Gorkunov, and K. H. Ringhofer, Phys. Rev. E 67, 065601 (2003).

${ }^{4}$ A. A. Zharov, I. V. Shadrivov, and Yu. S. Kivshar, Phys. Rev. Lett. 91, 037401 (2003).

${ }^{5}$ M. Lapine and M. Gorkunov, Phys. Rev. E 70, 066601 (2004).

${ }^{6}$ M. Gorkunov and M. Lapine, Phys. Rev. B 70, 235109 (2004).

${ }^{7}$ S. O'Brien, D. McPeake, S. A. Ramakrishna, and J. B. Pendry, Phys. Rev. B 69, 241101(R) (2004).

${ }^{8}$ N. A. Zharova, I. V. Shadrivov, A. A. Zharov, and Yu. S. Kivshar, Opt. Express 13, 1291 (2005).

${ }^{9}$ I. V. Shadrivov, A. A. Zharov, and Yu. S. Kivshar, J. Opt. Soc. Am. B 23 (in press).

${ }^{10}$ A. A. Zharov, N. A. Zharova, I. V. Shadrivov, and Yu. S. Kivshar, Appl. Phys. Lett. 87, 091104 (2005).

${ }^{11}$ I. V. Shadrivov and Yu. S. Kivshar, J. Opt. A, Pure Appl. Opt. 7, S68 (2005).

${ }^{12}$ H. Chen, L. Ran, J. Huangfu, X. Zhang, K. Chen, T. M. Grzegorczyk, and J. A. Kong, J. Appl. Phys. 96, 5338 (2004).

${ }^{13}$ O. Sydoruk, O. Zhuromskyy, E. Shamonina, and L. Solymar, Appl. Phys. Lett. 87, 072501 (2005).

${ }^{14}$ M. Gorkunov, M. Lapine, E. Shamonina, and K. H. Ringhofer, Eur. Phys. J. B 28, 263 (2002).

${ }^{15}$ R. Marques, F. Medina, and R. Rafii El Idrissi, Phys. Rev. B 65, 144440 (2002). 